\title{
Mortalidad tras un año de protocolización en el manejo del paciente quemado*
}

\author{
Drs. JORGE VILLEGAS C. ${ }^{1}$, ESTEBAN TORRES E. ${ }^{1}$, CÉSAR PEDREROS P. ${ }^{1}$, \\ EU PAULINA SINGH O. ${ }^{1}$, Dr. CRISTÓBAL LONGTON B. ${ }^{2}$, Int. JUAN CARLOS SAID S. ${ }^{3}$, \\ Drs. GABRIEL GARCÍA P. ${ }^{1}$, ISIDE BRAVO Y. ${ }^{1}$, SANDRA WHITTLE V. ${ }^{1}$, FERNANDA DEICHLER V. ${ }^{2}$
}

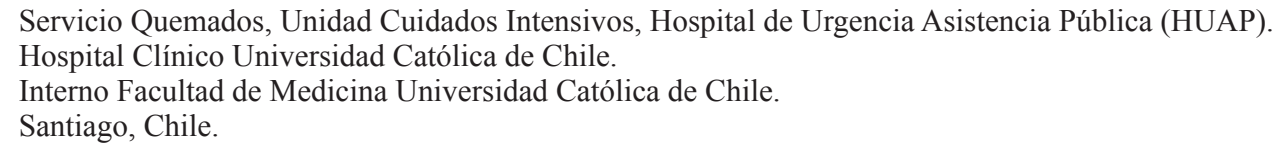

\section{Abstract}

\section{Effects of standarization of burn patient treatment on mortality}

Background: In 2006, the burn unit of an emergency public hospital in Chile standardized its medical and surgical treatment protocols. Aim: To analyze the evolution of mortality among patients admitted to the unit after the standardization process. Material and Methods: Patients admitted to the unit between March 2005 and March 2006, were analyzed. An early surgical debridement was performed, according to the guidelines, after a standardized reanimation with Lactate Ringer using Parkland formula ( $4 \mathrm{ml} / \mathrm{kg} / \%$ burn surface area). All patients were monitored. An algorithm was used to decide the indication of vasoactive drugs or invasive monitorization. Results: Eighty eight patients aged $43 \pm 19$ years (65\% males) were admitted. The estimated percentage body burned was $30 \pm 21 \%$, deep in $12.5 \pm 17 \%$. A mean of $9.122 \pm 6.930 \mathrm{ml}$ of fluid were administered in the first 24 hours. The first surgical debridement was performed at 48 hours (range 1 to 15 days). The first escharotomy was performed at 4.2 days (range 3 hours -15 days), the first covering at 3.8 days (range 3 hours-19 days) the first auto graft at 18 days (range 4-26 days). Operative times in the first three surgical procedures were less than $87 \mathrm{~min}$. Global mortality was $37 \%$. Conclusions: The standardized treatment of burns resulted in an absolute reduction of mortality.

Key words: Burns, standardized treatment, mortality.

\section{Resumen}

Introducción: En el 2005 el Servicio de Quemados del HUAP inició un proceso de modernización. Durante el primer año se intervino en el protocolo terapéutico médico y quirúrgico. Se analizarán la mortalidad observada en este período. Materiales y Métodos: Se analizaron los pacientes admitidos en Cuidados Intermedios e Intensivos de nuestra unidad desde el $1^{\circ}$ de marzo de 2005 a $1^{\circ}$ de marzo de 2006. Se realizó aseo quirúrgico precoz, según protocolo, previa reanimación estandarizada con Ringer Lactato usando la fórmula de Parkland, $4 \mathrm{cc} / \mathrm{kg} / \%$ SCT (superficie corporal total). Todos los casos fueron monitorizados, guiados por diuresis y con algoritmo para decidir inicio de drogas vasoactivas o monitorización invasiva.

*Recibido el 12 de Mayo de 2008 y aceptado para publicación el 5 de Noviembre de 2009.

Correspondencia: Dr. Jorge Villegas C.

Marchant Pereira 313, Oficina 101, Santiago, Chile.

E-mail: docvillegas@gmail.com 
Resultados: Ingresan 88 pacientes, $58 \%$ trasladados de otros centros. Edad promedio $43 \pm 18,9$ años; hombres $65 \%$, mujeres $35 \%$; superficie corporal quemada $30 \pm 21 \%$, profunda $12,5 \pm 17 \%$. Se administró en promedio $9.122 \pm 6.930 \mathrm{cc}$ durante las primeras $24 \mathrm{hrs}$. Primer aseo se realizó a las $48 \mathrm{hrs}(1 \mathrm{hr}-15 \mathrm{días}), 1^{\mathrm{a}}$ escarectomía a los 4,2 días ( 3 hrs - 15 días), $1^{\text {a }}$ cobertura a los 3,8 días ( 3 hrs - 19 días), 1er autoinjerto a los 18 días (4 - 26 días). Tiempos operatorios promedio en las 3 primeras cirugías inferiores a 87 min. Mortalidad absoluta disminuyó a 19\%. Mortalidad global 37\%. Conclusiones: Luego del primer año del proceso de modernización, con reanimación estandarizada, equipos quirúrgicos proporcionales a la SCT quemada con disminución de los tiempos quirúrgicos, asociado a un manejo multidisciplinario, se logró una disminución de la mortalidad global.

Palabras clave: Quemados, reanimación estandarizada.

\section{Introducción}

La sobrevida de los pacientes quemados críticos ha mejorado ostensiblemente en los últimos 30 años ${ }^{1,2}$, debido a una mejor reanimación, oportuna y protocolizada; introducción de escarectomía precoz y cobertura inmediata; soporte vital en terapia intensiva, nutrición enteral ${ }^{3} \mathrm{y}$, en menor medida al aporte de nuevos antibióticos en el tratamiento de la infección.

En el Hospital de Urgencia Asistencia Pública (HUAP) de Santiago, creado en 1967, está instalado el único Servicio especializado en el tratamiento de enfermos quemados críticos adultos en el sistema de salud público nacional. Este Servicio recibe los pacientes adultos que sufren quemaduras graves, derivados desde todo el país, con excepción de los accidentados del trabajo afiliados al Sistema de Mutuales de Seguridad. Si bien en el sistema de salud público la red de atención de los pacientes pediátricos está muy desarrollada, aquella destinada a los pacientes adultos se encuentra en etapas anteriores debido, entre otros, al menor impacto relativo de las quemaduras comparada con el conjunto de la patología quirúrgica y a la mayor complejidad de los pacientes adultos condicionada tanto por el mecanismo de la lesión como por la morbilidad asociada presente en la mayoría de los pacientes.

El servicio de quemados HUAP tiene un promedio de 270 ingresos anuales, de los cuales un 90\% son derivados de otros establecimientos hospitalarios del país. Hay una marcada variación estacional con un índice ocupacional de aproximadamente un $60 \%$ en verano y $100 \%$ en invierno.

La mortalidad global por causa de las quemaduras varía alrededor de $19 \%$, con una correlación directa con la edad, la superficie corporal quemada, y la profundidad, a lo que se agrega la presencia de injuria por inhalación (calor, partículas y gases tóxicos), morbilidad asociada y de sepsis bacteriana ${ }^{4}$. Es por eso que para análisis comparativos se continúa usando el "Índice de Garcés", el que se detalla más adelante.

En la última década, los índices de morbimortali- $\mathrm{dad}^{5}$ en los grupos de mayor gravedad se estancaron a pesar de los cambios introducidos en los años previos (Tabla 1). Debido a esto en Enero del año 2005 , se incorporó un experto ${ }^{6}$ y se inició un proceso de modernización, dirigido a intervenir el conjunto de los factores involucrados en los resultados del tratamiento:

1. Soporte Físico: Planta física, Sistemas de Circulación, Equipamiento e Insumos.

2. Recursos Humanos: Dotación, Capacitación.

3. Organización: Estructura Organizativa, Procesos, Protocolo Terapéutico.

Durante el año 2005 fue posible intervenir el protocolo terapéutico, incorporar insumos básicos, mejorar parcialmente procesos y avanzar en el desarrollo organizacional, generando instancias de discusión de pacientes y análisis sistemático de resultados, estableciendo indicadores de resultados susceptibles de evaluar prospectivamente. Se introdujeron nuevos esquemas de trabajo tanto en la reanimación como en el plan quirúrgico. Se incorporaron becados de cirugía en rotación de dos meses y cirujanos de trauma en estadías de perfeccionamiento de un año.

El objetivo de este trabajo es analizar el impacto logrado en la mortalidad global, con los cambios en el primer año de implementación del nuevo protocolo terapéutico médico y quirúrgico. Se usó como línea de base para comparar, los resultados de los años 2000 a 2004 del informe de Mortalidad por Grupos de Gravedad que elabora anualmente Informática Médica del HUAP.

\section{Material y Método}

Se analizaron los pacientes admitidos en cuidados Intermedios e intensivos de nuestro Servicio desde el $1^{\circ}$ de marzo de 2005 al $1^{\circ}$ de marzo de 2006, es decir, los quemados graves, críticos y de sobrevida excepcional.

El Servicio de Quemados del HUAP consta de un sector de cuidados intensivos/intermedios: dos salas de cuatro camas cada una y un aislamiento; un 
Tabla 1. Mortalidad. Quemados por Índice de Gravedad. HUAP*

\begin{tabular}{cccccc}
\hline Año & QSE (\%) & QCR (\%) & QGR (\%) & QMO (\%) & QLE (\%) \\
\hline 2000 & 93 & 70 & 20 & 4,8 & 0 \\
2001 & 95 & 76,6 & 25 & 3,8 & 0 \\
2002 & 100 & 86 & 35 & 3,6 & 0 \\
2003 & 86 & 73 & 24 & 4,9 & 0 \\
2004 & 95 & 55 & 26 & 3,2 & 3,2 \\
Total & 95 & 75 & 26 & 4 & 0,6 \\
\hline
\end{tabular}

QSE: quemado sobrevida excepcional, QCR: quemado crítico, QGR: quemado grave, QMO: quemado moderado, QLE: quemado leve. *Informática médica.

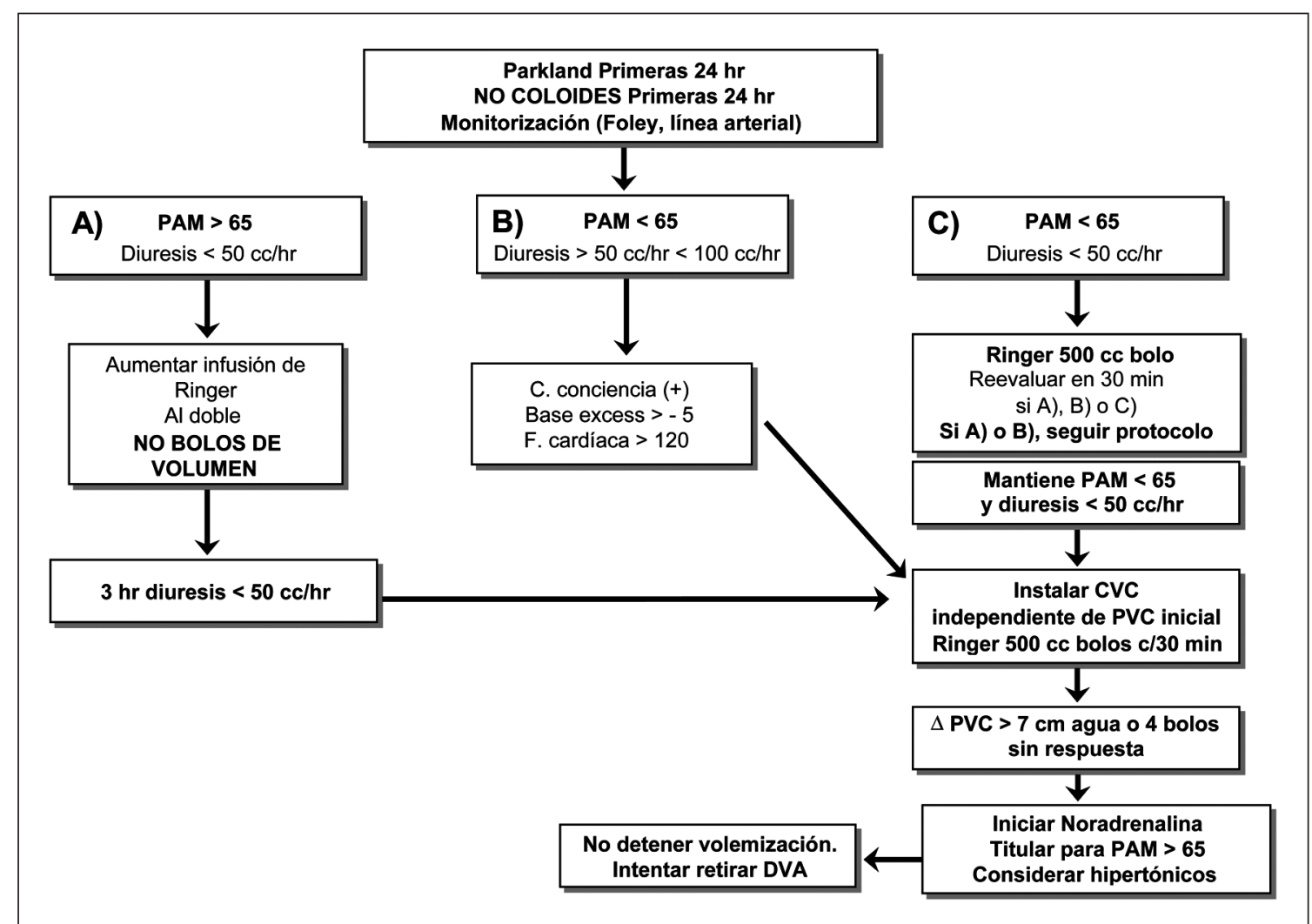

Figura 1. Algoritmo para reanimación de paciente quemado admitido en Cuidados Intermedios e Intensivos en el Servicio de Quemados del HUAP (Presentado en el XI Congreso Argentino de Quemaduras 2005, Buenos Aires y en el LXVII Congreso Chileno e Internacional de Cirugía 2005, Pucón).

pabellón quirúrgico exclusivo para la atención de pacientes quemados y un sector para pacientes leves y moderados de 15 camas. El equipo de trabajo está compuesto por 2 cirujanos plásticos experimentados y 3 cirujanos de otras especialidades con experiencia en manejo quirúrgico de pacientes quemados, 6 médicos internistas a cargo de cuidados intensivos, y relevos cada 12 horas de 2 enfermeras y 9 técnicos paramédicos en cada turno.

Los pacientes que ingresaron a dicho sector recibieron reanimación estandarizada (Figura 1) con Solución de Ringer Lactato (SRL) en base a fórmula de Parkland (4 cc/kg/\% SCT). En los pacientes con trauma asociado, injuria inhalatoria, quemadura 


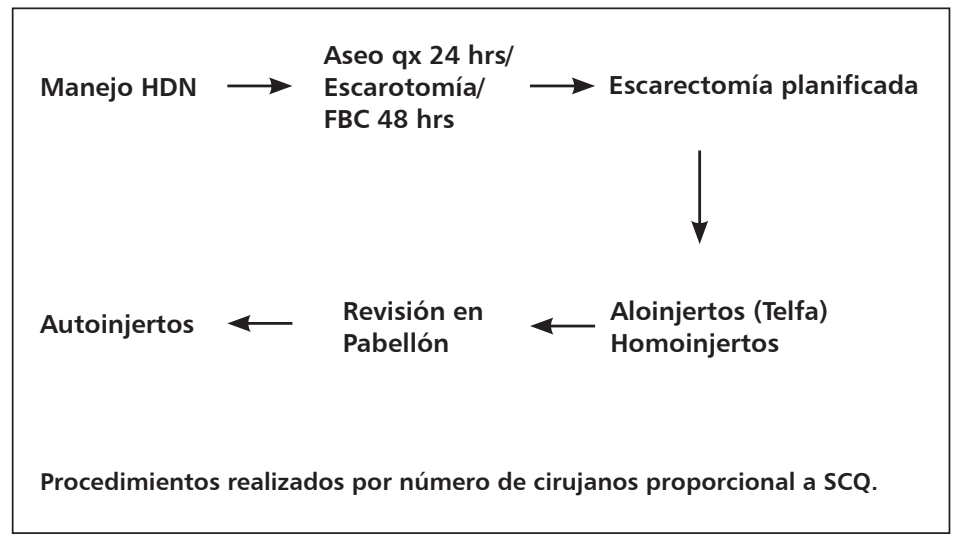

Figura 2. Protocolo quirúrgico para paciente quemado admitido en Cuidados Intermedios e Intensivos en el Servicio de Quemados del HUAP (Presentado en el XI Congreso Argentino de Quemaduras, Buenos Aires 2005 y en el LXVII Congreso Chileno e Internacional de Cirugía, Pucón 2005).

eléctrica o inicio tardío de la reanimación se utilizó un esquema de $5,6 \mathrm{cc} / \mathrm{kg} / \%$ SCT de SRL. Todos los pacientes fueron monitorizados, la efectividad del aporte de volumen se controló por medio de la diuresis y se aplicó un algoritmo para decidir el uso de drogas vasoactivas o el inicio de la monitorización invasiva.

De acuerdo a la evaluación clínica del equipo quirúrgico de la unidad, se realizó el primer aseo quirúrgico a la brevedad, planificando el resto de las cirugías según cada paciente de acuerdo al protocolo establecido sobre la base de dos principios: Protección de los remanentes epiteliales viables en las quemaduras de espesor parcial. Escarectomía precoz y cubierta inmediata en las de espesor total (Figura 2). Ante la sospecha de injuria inhalatoria, en casos seleccionados, se realizó fibrobroncoscopía para confirmar su existencia y evaluar la magnitud del daño. Se descartó la balneoterapia como procedimiento terapéutico porque se carece de condiciones estructurales para asegurar su realización en condiciones de asepsia quirúrgica. No se utilizó terapia antiséptica o antibiótica tópica sin un diagnóstico preciso que la indicara. Los procedimientos destinados a tratar la herida se hicieron en quirófano, con técnica quirúrgica y anestesia general. La anestesia regional fue de uso excepcional.

Los datos registrados de los pacientes fueron: variables demográficas (sexo, edad, IMC); variables relacionadas con las características de la quemadura (superficie total quemada, superficie quemada profunda, latencia en horas hasta la primera cirugía, latencia en días hasta el primer injerto, número total de cirugías); y variables clínicas (volumen recibido en reanimación, días de drogas vasoactivas, de ventilación mecánica, injuria inhalatoria y mortalidad por grupo de gravedad) (Tabla 2).

El análisis estadístico se realizó con el software SPSS 12.0. Los resultados fueron expresados como
Tabla 2. Índice de Gravedad de Garcés

\begin{tabular}{rlc}
\hline \multicolumn{1}{l}{ Índice } & Grupo & \% Mortalidad \\
\hline $0-40$ & Leve & Excepcional \\
$41-70$ & Moderado & 5 \\
$71-100$ & Grave & 30 \\
$101-150$ & Crítico & 75 \\
$>150$ & Sobrevida excepcional & 99 \\
\hline
\end{tabular}

IG: \% SCQ A x $1+\%$ SCQ AB x 2+\% SCQ B x $3+$ Edad (mínimo 20 años). IG: índice gravedad, SCQ: superficie corporal quemada, A-AB-B: profundidad de quemadura.

medias estadísticas con las respectivas desviaciones estándar. Se consideró la significancia estadística con un valor $\mathrm{p}$ menor a 0,05 .

\section{Resultados}

En el período indicado, fueron admitidos 88 pacientes en la unidad de cuidados intermedios e intensivos de nuestro servicio ( $42 \%$ fueron ingresados directamente en el HUAP y $58 \%$ fueron trasladados desde otros centros (Tabla 3).

a. Variables demográficas: La edad promedio fue $43 \pm 18,9$ años, la distribución por sexos fue hombres $65 \%$, mujeres $35 \%$, con IMC $27 \pm 3,7$ y un peso promedio de $72,1 \pm 11,8 \mathrm{~kg}$.

b. Variables relacionadas con las características de la quemadura: El porcentaje de superficie total quemada fue $30 \pm 21 \%$, superficie profunda $12,5 \pm$ $17 \%$. El primer aseo quirúrgico se realizó en promedio a las $48 \mathrm{hrs}$ ( $1 \mathrm{hr}-15$ días), la primera escarectomía a los 4,2 días promedio ( $3 \mathrm{hrs}$ - 15 días), la primera cobertura a los 3,8 días promedio ( $3 \mathrm{hrs}-19$ 
Tabla 3. Origen de traslado paciente quemado

\begin{tabular}{lcc}
\hline & n & Porcentaje \\
\hline HUAP & 16 & 39 \\
Sótero del Río & 7 & 17,1 \\
Barros Luco & 3 & 7,3 \\
Coyhaique & 2 & 4,9 \\
San Juan de Dios & 2 & 4,9 \\
Mutual de Seguridad & 1 & 2,4 \\
San Bernardo & 1 & 2,4 \\
San José & 1 & 2,4 \\
Puerto Montt & 1 & 2,4 \\
Curacaví & 1 & 2,4 \\
H. el Pino & 1 & 2,4 \\
Talca & 1 & 2,4 \\
H. Salvador & 1 & 2,4 \\
G. Fricke & 1 & 2,4 \\
H. San Felipe & 1 & 2,4 \\
Concepción & 1 & 2,4 \\
\hline & & \\
\hline
\end{tabular}

días) y el primer autoinjerto a los 18 días promedio (4 - 26 días). El número total de cirugías fue de 5,16 en promedio ( 0 a 17$)$, con un aumento en el número total de cirugías del servicio de 42,7\% (508 en 2004 a 725 en 2005). El número de cirujanos es directamente proporcional al \% SCT, con tiempos operatorios promedio en las 3 primeras cirugías inferiores a 87 minutos (Tabla 4).

c. Variables clínicas: Los pacientes recibieron en promedio $9.122 \pm 6.930 \mathrm{cc}$ en las primeras $24 \mathrm{hrs}$, lo que representa un $105,4 \%$ del teórico. Se utilizaron vasopresores en promedio luego de 4,34 días postingreso ( 0 a 16), durante 4,95 días ( 0 a 33 ). El $40 \%$ de los pacientes presentó injuria inhalatoria. El promedio de días de ventilación mecánica fue de 8 (0 - 72). El tiempo de estadía en la unidad de pacien- te crítico fue de 19 días (1 a 98) y permanecieron hospitalizados un promedio de 34 días (1 a 165). La mortalidad global fue de $37 \%$.

\section{Discusión}

En nuestro país es posible distinguir epidemiológicamente tres grupos de pacientes quemados graves con perfiles claramente diferentes: niño accidentado del hogar, adulto accidentado del trabajo y adulto lesionado en el hogar o la vía pública ${ }^{5-7}$. Nuestros pacientes corresponden mayoritariamente al tercero de estos grupos. Estos se caracterizan por lesionarse principalmente con fuego, habitualmente son ancianos o adultos jóvenes en riesgo social, con un grado variable de deterioro en sus funciones cognitivas, ya sea por deterioro senil, drogadicción, alcoholismo ${ }^{8}$, o patología siquiátrica ${ }^{9}$, (con un $20 \%$ de lesiones autoinferidas (Bonzo). Por lo anterior la mayoría no está en condiciones de anticipar el riesgo, disminuir el daño o buscar ayuda al momento de lesionarse. Además son pacientes con escasa o nula red de apoyo social.

Es importante tener presente que la pérdida de la cubierta cutánea conlleva al deterioro de la capacidad de termorregular. Sin piel, el paciente pierde los mecanismos de control y adicionalmente pierde temperatura por radiación con mayor facilidad. Con la incorporación del nuevo protocolo quirúrgico (Figura 2), estandarización de la técnica quirúrgica y equipos de dos cirujanos o más, según la gravedad del enfermo, logramos disminuir los tiempos operatorios, minimizando el impacto de la cirugía y disminuyendo de forma considerable la hipotermia y sus complicaciones.

Sin embargo, las limitaciones del soporte físico, especialmente la carencia de aislamientos equipados y la escasa dotación de personal no nos permite aun el manejo óptimo de todos los aspectos mencionados e impide dar respuesta oportuna a todas las solicitudes de traslado.

Durante los últimos años, las cifras de mortalidad se habían mantenido sin mayores modificaciones y

Tabla 4. Número de cirujanos según SCT quemada

\begin{tabular}{|c|c|c|c|c|c|c|c|}
\hline & & & $\begin{array}{c}n \\
\text { cirujanos } 1^{\circ}\end{array}$ & $\begin{array}{c}n \\
\text { cirujanos } 2^{\circ}\end{array}$ & $\frac{n}{\text { cirujanos }} 3^{o}$ & $\begin{array}{c}\mathbf{n} \\
\text { cirujanos }\end{array}$ & $\begin{array}{c}\text { n } \\
\text { cirujanos }\end{array}$ \\
\hline Promedio & & 32,9 & 2,0 & 2,3 & 2,2 & 2,3 & 2,1 \\
\hline \multirow[t]{3}{*}{ Percentiles } & 25 & 14,0 & 2,0 & 2,0 & 1,3 & 2,0 & 1,0 \\
\hline & 50 & 33,0 & 2,0 & 2,0 & 2,0 & 2,0 & 2,0 \\
\hline & 75 & 43,5 & 2,3 & 3,0 & 3,0 & 3,0 & 3,0 \\
\hline
\end{tabular}




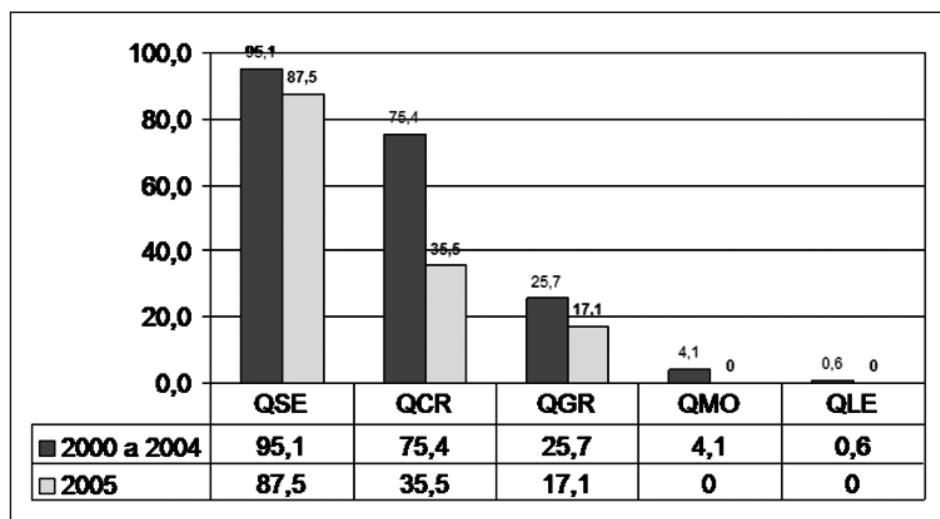

Figura 3. Mortalidad años 2000 a 2004 comparado con 2005 según Índice de Gravedad. QSE: quemado sobrevida excepcional. QCR: quemado crítico. QGR: quemado grave. QMO: quemado moderado. QLE: quemado leve. por encima de los estándares nacionales aceptados en la actualidad ${ }^{10}$; luego del primer año del proceso de modernización, hemos logrado una disminución de la mortalidad global y por grupo específico. El mayor impacto se observa en el Grupo Crítico (Figura 3). En el Grupo de Sobrevida Excepcional se acumulan aquellos pacientes irrescatables que fallecen en las primeras horas. Es necesario un análisis especial que establezca un límite de inclusión en el estudio. Igualmente es necesario incorporar en los Índices de Gravedad el peso de la injuria inhalatoria, el traumatismo asociado, el daño por energía eléctrica de alta tensión y la comorbilidad concomitante.

Aún podemos mejorar el cumplimiento de los plazos de este nuevo protocolo. Están pendientes las tareas de introducir las modificaciones necesarias en el área de soporte físico y recursos humanos y articular la red de atención para pacientes quemados adultos en el sistema público nacional, homogenizando protocolos y creando condiciones para traslados oportunos eficaces y eficientes.

\section{Referencias}

1. Huang Y, Yang Z. Measures for preventing early postburn damage improve survival rate of burn patients. Burns 2004; 30: 808-812.

2. Sagit Meshulam-Derazon. Prediction of Morbidity and Mortality on Admission to a Burn Unit. Plast Reconstr
Surg 2006; 118: 116-120.

3. Hart DW, Wolf SE, Chinkes DL, Beauford RB, Mlcak RP, Heggers JP, et al. Effects of Early Excision and Aggressive Enteral Feeding on Hypermetabolism, Catabolism, and Sepsis after Severe Burn. J Trauma 2003; 54: 755-764.

4. Pruitt BA Jr, McManus AT, Kim SH, Goodwin CW. Burn Wound Infections: Current Status. World J Surg 1998; 22: 135-145.

5. Villegas J. Quemaduras. Procedimientos habituales en el Tratamiento. Unidad de Quemados. Hospital Luis Calvo Mackenna.

6. Mejoramiento de la calidad de atención en el Servicio clínico de quemados del Hospital de Urgencia Asistencia Pública. Archivos Subdirección Administrativa. Hospital de Urgencia Asistencia Pública, Junio 2005.

7. Forjuoh SN. Burns in low -and middle- income countries: A review of available literature on descriptive epidemiology, risk factors, treatment, and prevention. Burns 2006; 32: 529-537.

8. Kavanaugha MJ, Clark C, Goto M, Kovacs EJ, Gamelli RL, Sayeed MM, et al. Effect of acute alcohol ingestion prior to burn injury on intestinal bacterial growth and barrier function. Burns 2005; 31: 290-296.

9. Tarrier N, Gregg L, Edwards J, Dunn K. The influence of pre-existing psychiatric illness on recovery in burn injury patients: the impact of psychosis and depression. Burns 2005; 31: 45-49.

10. Ministerio de salud. Guía Clínica Gran Quemado. Minsal 2007. 